\title{
2-Amino-6-Chlorobenzoic Acid Dissolved in Numerous Individual Solvents: Equilibrium Solubility, Thermodynamic Modelling and Mixing Properties
}

\author{
Yuxin Bao ${ }^{a}$, Renjie Xu ${ }^{b}$, Hongkun Zhao ${ }^{a,}$ \\ ${ }^{a}$ College of Chemistry \& Chemical Engineering, YangZhou University, YangZhou, Jiangsu 225002, People's \\ Republic of China \\ b Guangling college, YangZhou University, YangZhou, Jiangsu 225009, People's Republic of China
}

Corresponding author. Tel: + 8651487975568 ; Fax: + 8651487975244 .

*E-mail address: hkzhao@yzu.edu.cn (H.K. Zhao). 


\section{Computational process of the relative standard uncertainty of the determinations in mole fraction}

(1) Calculating the arithmetic mean value by the original data of measurement

$$
\mu=\frac{1}{n} \sum_{\mathrm{i}=1}^{n} x_{\mathrm{i}}
$$

here $n$ is number of measurements.

\section{(2) Calculating the standard deviation}

The uncertainty of measurement results generally contains several components. These components can be divided into two classes: class A and class B. Class A, which is obtained by Eq. (S2), refers to the repeatedly measured results in statistical method to calculate the standard deviation.

$$
u_{\mathrm{A}}=\frac{\sqrt{\frac{\sum_{\mathrm{i}=1}^{\mathrm{n}}\left(x_{\mathrm{i}}-\mu\right)^{2}}{n-1}}}{\sqrt{n}}
$$

Class B refers to the instrument error of estimation method to assess. When the measured values deviate to one direction with constant deviation due to error influence, then the uncertainty cannot be evaluated by using the statistical method. This kind of uncertainty is said to class B evaluated through Eq. (S3).

$$
u_{\mathrm{B}}=\frac{\Delta}{\sqrt{3}}
$$

where $\Delta$ stands for limit error or allowable error.

The liquid chromatographic measurement is performed to acquire the mole fraction of solute in equilibrium liquid phase. The UV standard curve is constructed before experiment. After sampling the liquid phase, the uncertainty is calculated with the calibration curve by using Eq. (S4).

$$
u_{\mathrm{c}}=\sqrt{u_{\mathrm{A}}^{2}+u_{\mathrm{B}}^{2}}
$$


(3) Calculating the relative standard deviation

The relative standard uncertainty is calculated with

$$
u_{x}=\frac{u_{\mathrm{c}}}{\bar{x}}
$$




\section{TABLE S1}

The computed values for $\Delta_{\text {mix }} G, \Delta_{\text {mix }} H, \Delta_{\text {mix }} S, \ln \gamma_{1}^{\infty}$ and $H_{1}^{E, \infty}$. ${ }^{a}$

\begin{tabular}{|c|c|c|c|c|c|}
\hline \multirow{2}{*}{$T / \mathrm{K}$} & $\Delta_{\operatorname{mix}} G$ & $\Delta_{\text {mix }} H$ & $\Delta_{\text {mix }} S$ & \multirow{2}{*}{$\ln \gamma_{1}^{\infty}$} & $H_{1}^{E, \infty}$ \\
\hline & $\mathrm{J} \cdot \mathrm{mol}^{-1}$ & $\mathrm{~J} \cdot \mathrm{mol}^{-1}$ & $\mathrm{~J} \cdot \mathrm{mol}^{-1} \cdot \mathrm{K}^{-1}$ & & $\mathrm{~kJ} \cdot \mathrm{mol}^{-1}$ \\
\hline \multicolumn{6}{|c|}{ ethanol } \\
\hline 278.15 & -329.7 & -32.50 & 1.069 & -0.487 & -1.057 \\
\hline 283.15 & -391.7 & -38.89 & 1.246 & -0.478 & \\
\hline 288.15 & -453.0 & -45.08 & 1.416 & -0.470 & \\
\hline 293.15 & -529.6 & -53.13 & 1.625 & -0.462 & \\
\hline 298.15 & -613.9 & -62.12 & 1.851 & -0.455 & \\
\hline 303.15 & -715.3 & -73.36 & 2.117 & -0.448 & \\
\hline 308.15 & -820.8 & -85.19 & 2.387 & -0.441 & \\
\hline 313.15 & -951.4 & -100.7 & 2.716 & -0.435 & \\
\hline 318.15 & -1088 & -117.4 & 3.051 & -0.429 & \\
\hline 323.15 & -1231 & -135.5 & 3.392 & -0.423 & \\
\hline \multicolumn{6}{|c|}{$n$-propanol } \\
\hline 278.15 & -234.1 & -15.70 & 0.785 & -0.100 & -0.694 \\
\hline 283.15 & -278.6 & -18.57 & 0.918 & -0.094 & \\
\hline 288.15 & -327.8 & -21.70 & 1.062 & -0.089 & \\
\hline 293.15 & -379.2 & -24.87 & 1.209 & -0.084 & \\
\hline 298.15 & -436.4 & -28.34 & 1.369 & -0.079 & \\
\hline 303.15 & -508.2 & -32.85 & 1.568 & -0.074 & \\
\hline 308.15 & -585.7 & -37.63 & 1.779 & -0.070 & \\
\hline 313.15 & -684.1 & -43.94 & 2.044 & -0.066 & \\
\hline 318.15 & -788.0 & -50.50 & 2.318 & -0.062 & \\
\hline 323.15 & -904.8 & -57.89 & 2.621 & -0.058 & \\
\hline \multicolumn{6}{|c|}{ isopropanol } \\
\hline 278.15 & -224.0 & -11.58 & 0.764 & -0.038 & -0.528 \\
\hline 283.15 & -260.1 & -13.21 & 0.872 & -0.034 & \\
\hline 288.15 & -304.6 & -15.23 & 1.004 & -0.030 & \\
\hline 293.15 & -356.9 & -17.61 & 1.157 & -0.026 & \\
\hline 298.15 & -417.7 & -20.35 & 1.333 & -0.022 & \\
\hline 303.15 & -486.6 & -23.40 & 1.528 & -0.018 & \\
\hline 308.15 & -562.7 & -26.70 & 1.740 & -0.015 & \\
\hline 313.15 & -645.9 & -30.19 & 1.966 & -0.012 & \\
\hline 318.15 & -751.6 & -34.79 & 2.253 & -0.009 & \\
\hline 323.15 & -864.5 & -39.54 & 2.553 & -0.007 & \\
\hline
\end{tabular}




\begin{tabular}{|c|c|c|c|c|c|}
\hline \multicolumn{6}{|c|}{ EG } \\
\hline 278.15 & -155.4 & 18.48 & 0.625 & 0.286 & 1.469 \\
\hline 283.15 & -196.8 & 23.81 & 0.779 & 0.275 & \\
\hline 288.15 & -245.0 & 30.05 & 0.955 & 0.264 & \\
\hline 293.15 & -298.6 & 36.93 & 1.144 & 0.254 & \\
\hline 298.15 & -357.6 & 44.42 & 1.348 & 0.244 & \\
\hline 303.15 & -421.7 & 52.39 & 1.564 & 0.234 & \\
\hline 308.15 & -490.3 & 60.70 & 1.788 & 0.224 & \\
\hline 313.15 & -567.1 & 69.76 & 2.034 & 0.215 & \\
\hline 318.15 & -651.2 & 79.32 & 2.296 & 0.206 & \\
\hline 323.15 & -751.2 & 90.29 & 2.604 & 0.198 & \\
\hline \multicolumn{6}{|c|}{ ethyl acetate } \\
\hline 278.15 & -295.1 & -26.01 & 0.967 & -0.324 & -0.990 \\
\hline 283.15 & -341.7 & -30.33 & 1.100 & -0.316 & \\
\hline 288.15 & -397.3 & -35.63 & 1.255 & -0.309 & \\
\hline 293.15 & -457.4 & -41.41 & 1.419 & -0.301 & \\
\hline 298.15 & -535.7 & -49.32 & 1.631 & -0.295 & \\
\hline 303.15 & -623.2 & -58.39 & 1.863 & -0.288 & \\
\hline 308.15 & -720.3 & -68.72 & 2.115 & -0.282 & \\
\hline 313.15 & -826.4 & -80.31 & 2.383 & -0.276 & \\
\hline 318.15 & -946.7 & -93.95 & 2.680 & -0.270 & \\
\hline 323.15 & -1077 & -109.2 & 2.994 & -0.264 & \\
\hline \multicolumn{6}{|c|}{ acetonitrile } \\
\hline 278.15 & -87.24 & 20.00 & 0.386 & 0.858 & 2.770 \\
\hline 283.15 & -111.9 & 26.42 & 0.488 & 0.837 & \\
\hline 288.15 & -141.2 & 34.30 & 0.609 & 0.817 & \\
\hline 293.15 & -175.2 & 43.66 & 0.747 & 0.797 & \\
\hline 298.15 & -213.2 & 54.31 & 0.897 & 0.778 & \\
\hline 303.15 & -256.5 & 66.60 & 1.066 & 0.760 & \\
\hline 308.15 & -302.1 & 79.58 & 1.239 & 0.742 & \\
\hline 313.15 & -354.4 & 94.59 & 1.434 & 0.724 & \\
\hline 318.15 & -411.3 & 110.93 & 1.642 & 0.708 & \\
\hline 323.15 & -479.1 & 130.50 & 1.886 & 0.691 & \\
\hline \multicolumn{6}{|c|}{$n$-butanol } \\
\hline 278.15 & -195.9 & -19.61 & 0.634 & 0.157 & -0.729 \\
\hline 283.15 & -232.4 & -23.02 & 0.740 & 0.164 & \\
\hline 288.15 & -271.2 & -26.53 & 0.849 & 0.171 & \\
\hline 293.15 & -319.6 & -31.22 & 0.984 & 0.177 & \\
\hline 298.15 & -377.3 & -37.12 & 1.141 & 0.182 & \\
\hline
\end{tabular}




\begin{tabular}{|c|c|c|c|c|c|}
\hline 303.15 & -441.3 & -43.82 & 1.311 & 0.187 & \\
\hline 308.15 & -515.6 & -52.03 & 1.504 & 0.191 & \\
\hline 313.15 & -603.2 & -62.36 & 1.727 & 0.195 & \\
\hline 318.15 & -708.1 & -75.66 & 1.988 & 0.198 & \\
\hline 323.15 & -826.1 & -91.39 & 2.274 & 0.201 & \\
\hline \multicolumn{6}{|c|}{ isobutanol } \\
\hline 278.15 & -120.7 & 34.09 & 0.547 & 0.744 & 3.282 \\
\hline 283.15 & -158.2 & 46.02 & 0.709 & 0.719 & \\
\hline 288.15 & -199.4 & 59.28 & 0.882 & 0.695 & \\
\hline 293.15 & -248.1 & 75.09 & 1.084 & 0.672 & \\
\hline 298.15 & -303.2 & 92.99 & 1.307 & 0.649 & \\
\hline 303.15 & -365.8 & 113.21 & 1.555 & 0.627 & \\
\hline 308.15 & -433.8 & 134.75 & 1.815 & 0.606 & \\
\hline 313.15 & -509.9 & 158.39 & 2.101 & 0.586 & \\
\hline 318.15 & -589.6 & 182.28 & 2.389 & 0.566 & \\
\hline 323.15 & -683.2 & 209.58 & 2.721 & 0.546 & \\
\hline \multicolumn{6}{|c|}{$n$-heptanol } \\
\hline 278.15 & -112.3 & -42.75 & 0.250 & 0.857 & -2.066 \\
\hline 283.15 & -146.5 & -57.94 & 0.313 & 0.879 & \\
\hline 288.15 & -185.5 & -75.91 & 0.380 & 0.899 & \\
\hline 293.15 & -227.4 & -95.39 & 0.450 & 0.917 & \\
\hline 298.15 & -270.9 & -115.43 & 0.522 & 0.932 & \\
\hline 303.15 & -321.7 & -139.59 & 0.601 & 0.945 & \\
\hline 308.15 & -381.2 & -168.96 & 0.689 & 0.957 & \\
\hline 313.15 & -450.5 & -204.11 & 0.787 & 0.966 & \\
\hline 318.15 & -534.9 & -248.86 & 0.899 & 0.975 & \\
\hline 323.15 & -636.9 & -304.55 & 1.028 & 0.982 & \\
\hline \multicolumn{6}{|c|}{ water } \\
\hline 278.15 & -6.451 & 0.949 & 0.027 & 3.589 & 1.677 \\
\hline 283.15 & -7.510 & 1.120 & 0.030 & 3.576 & \\
\hline 288.15 & -8.78 & 1.328 & 0.035 & 3.564 & \\
\hline 293.15 & -10.36 & 1.597 & 0.041 & 3.552 & \\
\hline 298.15 & -12.15 & 1.912 & 0.047 & 3.540 & \\
\hline 303.15 & -14.29 & 2.299 & 0.055 & 3.529 & \\
\hline 308.15 & -16.78 & 2.765 & 0.063 & 3.518 & \\
\hline 313.15 & -19.66 & 3.325 & 0.073 & 3.508 & \\
\hline 318.15 & -23.11 & 4.024 & 0.085 & 3.498 & \\
\hline 323.15 & -26.83 & 4.802 & 0.098 & 3.488 & \\
\hline \multicolumn{6}{|c|}{ 1-Octanol } \\
\hline 278.15 & -96.43 & -43.98 & 0.189 & 1.089 & -2.198 \\
\hline
\end{tabular}




\begin{tabular}{|c|c|c|c|c|c|}
\hline 283.15 & -127.3 & -60.76 & 0.235 & 1.114 & \\
\hline 288.15 & -159.9 & -78.68 & 0.282 & 1.135 & \\
\hline 293.15 & -195.0 & -98.02 & 0.331 & 1.154 & \\
\hline 298.15 & -234.1 & -119.7 & 0.383 & 1.171 & \\
\hline 303.15 & -283.5 & -149.2 & 0.443 & 1.185 & \\
\hline 308.15 & -341.2 & -184.6 & 0.508 & 1.197 & \\
\hline 313.15 & -415.2 & -232.6 & 0.583 & 1.207 & \\
\hline 318.15 & -506.2 & -293.7 & 0.668 & 1.215 & \\
\hline 323.15 & -604.4 & -358.8 & 0.760 & 1.222 & \\
\hline \multicolumn{6}{|c|}{ toluene } \\
\hline 278.15 & -50.48 & 4.463 & 0.198 & 1.522 & 1.011 \\
\hline 283.15 & -57.64 & 5.143 & 0.222 & 1.514 & \\
\hline 288.15 & -67.71 & 6.149 & 0.256 & 1.507 & \\
\hline 293.15 & -80.39 & 7.468 & 0.300 & 1.499 & \\
\hline 298.15 & -96.14 & 9.176 & 0.353 & 1.492 & \\
\hline 303.15 & -112.9 & 11.04 & 0.409 & 1.486 & \\
\hline 308.15 & -131.0 & 13.10 & 0.468 & 1.479 & \\
\hline 313.15 & -150.0 & 15.32 & 0.528 & 1.473 & \\
\hline 318.15 & -168.9 & 17.55 & 0.586 & 1.467 & \\
\hline 323.15 & -190.9 & 20.23 & 0.653 & 1.461 & \\
\hline \multicolumn{6}{|c|}{ cyclohexane } \\
\hline 283.15 & -0.465 & 0.588 & 0.0037 & 6.269 & 14.717 \\
\hline 288.15 & -0.655 & 0.856 & 0.0053 & 6.160 & \\
\hline 293.15 & -0.917 & 1.241 & 0.0074 & 6.056 & \\
\hline 298.15 & -1.185 & 1.631 & 0.0094 & 5.954 & \\
\hline 303.15 & -1.551 & 2.185 & 0.012 & 5.856 & \\
\hline 308.15 & -1.986 & 2.851 & 0.016 & 5.762 & \\
\hline 313.15 & -2.537 & 3.716 & 0.02 & 5.670 & \\
\hline 318.15 & -3.162 & 4.699 & 0.025 & 5.581 & \\
\hline 323.15 & -3.965 & 5.993 & 0.031 & 5.495 & \\
\hline \multicolumn{6}{|c|}{ NMP } \\
\hline 278.15 & -873.1 & -212.94 & 2.373 & -1.787 & -3.395 \\
\hline 283.15 & -931.7 & -224.58 & 2.498 & -1.760 & \\
\hline 288.15 & -1009 & -240.65 & 2.667 & -1.735 & \\
\hline 293.15 & -1093 & -257.98 & 2.849 & -1.711 & \\
\hline 298.15 & -1192 & -278.45 & 3.065 & -1.687 & \\
\hline 303.15 & -1304 & -301.48 & 3.308 & -1.665 & \\
\hline 308.15 & -1436 & -328.23 & 3.594 & -1.643 & \\
\hline 313.15 & -1591 & -359.32 & 3.932 & -1.622 & \\
\hline 318.15 & -1750 & -389.87 & 4.276 & -1.602 & \\
\hline
\end{tabular}




\begin{tabular}{lllll}
\hline 323.15 & -1920 & -420.72 & 4.639 & -1.582 \\
\hline
\end{tabular}

${ }^{a} \Delta_{\text {mix }} G, \Delta_{\text {mix }} H$ and $\Delta_{\text {mix }} S$ signify, respectively, the mixing Gibbs free energy, mixing enthalpy, and mixing entropy; $\gamma_{1}^{\infty}$ refers to the activity coefficient under infinitesimal concentration; and $H_{1}^{E, \infty}$ signifies reduced excess enthalpy under infinitesimal concentration. Standard uncertainties $u$ are $u\left(\Delta_{\text {mix }} G\right)=u\left(\Delta_{\text {mix }} H\right)=0.3 \mathrm{~J} \cdot \mathrm{mol}^{-1}$, $u\left(\Delta_{\mathrm{mix}} S\right)=0.002 \mathrm{~J} \cdot \mathrm{mol}^{-1} \cdot \mathrm{K}^{-1}$. 